\title{
Müssen Preisgelder versteuert werden? In der Regel ja!
}

\section{Zusammenfassung}

So groß die Freude der Preisträger über die innen zuteil gewordene Auszeichnung ihrer Leistungen und die Zuerkennung der damit häufig verbundenen Preisgelder zunächst ist, umso ernüchternder ist oftmals der Zugriff des Finanzamts auf den finanziellen Zuverdienst: Die Finanzämter ordnen nämlich das Preisgeld regelmäßig den Einkünften aus nichtselbst-ändiger Arbeit zu und sehen es als steuerpflichtige Einnahme an. Immer wieder ist die Steuerbarkeit von Preisgeldern bereits Gegenstand finanzgerichtlicher Auseinandersetzungen gewesen und meist ist die Ansicht der Finanzämter durch die Gerichte auch bestätigt worden. Verbände, Stiftungen oder wissenschaftliche Fachgesellschaften, die Preise verleihen, nehmen mit dem Inhalt der Ausschreibung und Auslobung der Preise einen gewissen Einfluss auf die Steuerbarkeit, sollten die (künftigen) Preisträger aber regelmäßig darauf hinweisen, dass unter Umständen eine Steuerpflicht der Preisträger für die Zuerkennung der mit den Preisen verbundenen Dotationen besteht.

\author{
Linda Kuball ${ }^{1}$ \\ Albrecht Wienke ${ }^{2}$ \\ 1 Kanzlei WBK \\ 2 Wienke \& Becker - Köln
}

\section{Text}

1. Grundsätzlich können Preise der Besteuerung als Einnahme in Geld im Sinne von § 8 Abs. 1 des Einkommensteuergesetzes (EStG) unterliegen, die dem Preisträger im Rahmen der Einkunftsart nach § 2 Abs. $1 \mathrm{Satz} 1 \mathrm{Nr}$. 4 EStG bei seinen Einkünften aus unselbständiger Arbeit gemäß § 19 Abs. 1 Satz 1 Nr. 4 i.V.m. Satz 2 EStG zufließen.

Nach § 19 Abs. 1 Satz 1 Nr. 1 EStG gehören zu den Einkünften aus nichtselbständiger Tätigkeit unter anderem auch Vorteile für eine Beschäftigung im öffentlichen oder privaten Dienst. Vorteile werden „für“ eine Beschäftigung gewährt, wenn sie durch das Dienstverhältnis veranlasst sind. Das ist der Fall, wenn der Vorteil mit Rücksicht auf das Dienstverhältnis eingeräumt wird und sich der Preis im weitesten Sinne als Gegenleistung für das Zurverfügungstellen der individuellen Arbeitskraft des Beschäftigten erweist bzw. wenn die Zuwendung sich als Frucht der Arbeit darstellt und im Zusammenhang mit dem Dienstverhältnis steht. Dies gilt auch, wenn die Zuwendung durch einen Dritten, also nicht direkt durch den Arbeitgeber, erfolgt.

Der einem Arbeitnehmer von seinem Arbeitgeber oder von einem Dritten verliehene Preis führt daher beim Preisträger regelmäßig zu steuerpflichtigen Einkünften aus nichtselbständiger Tätigkeit, wenn die Preisverleihung nicht vor allem eine Ehrung der Persönlichkeit des Preisträgers darstellt, sondern wirtschaftlich den Charakter eines leistungsbezogenen Entgelts hat und einen wirtschaftlichen Bezug zu der Tätigkeit des Preisträgers aufweist. Ob eher die berufliche Leistung des Preisträgers geehrt wird oder vornehmlich seine Persönlichkeit, sein Gesamtschaffen oder sein Lebens- werk, bemisst sich nach den jeweiligen Gesamtumständen des Einzelfalls. Dass ein sachlicher und persönlicher äußerlicher Zusammenhang zwischen dem Preisgeld und der Arbeitnehmertätigkeit besteht, ist unschädlich, solange dieser nicht die Intensität eines ertragsteuerlich relevanten wirtschaftlichen Leistungsaustauschs erreicht.

Für ein leistungsbezogenes steuerpflichtiges Entgelt sprechen z.B.

- die Verleihung des Preises für besondere Leistungen, Innovationen, Ablaufverbesserungen, Veranstaltungen oder realisierte Projekte in den von dem Preisträgern wahrgenommenen Aufgabenbereichen in ihrer typischen beruflichen Tätigkeit oder

- bei einem Wissenschaftspreis die konkret ausgezeichneten Leistungen im Rahmen der Aufgaben eines Wissenschaftlers, zu dessen Aufgaben u.a. die Lehre und die Vermittlung des Fachwissens an Studierende gehört.

Ein Preisgeld kann auch dann eine leistungsbezogene steuerpflichtige Einnahme sein, wenn die ausgezeichnete Leistung bereits vor einer Auslobung oder unabhängig von einer möglichen Preisverleihung erbracht wurde oder wenn keine gesonderten Bewerbungen notwendig sind, sondern nur Kandidaten von Dritten vorgeschlagen werden können.

2. Kein steuerpflichtiger Arbeitslohn liegt demgegenüber vor, wenn die mit dem Preis verbundene finanzielle Zuwendung aufgrund sonstiger, nicht auf dem Dienstverhältnis beruhender Beziehungen gewährt wird. Demnach sind als persönlich veranlasste Preise solche anzusehen, die für das Lebenswerk oder das Gesamtschaffen des Preisträgers verliehen werden 
bzw. die die Persönlichkeit oder Grundhaltung des Empfängers ehren und seine Vorbildfunktion herausstellen sollen. Solchen Preisverleihungen liegt regelmäßig kein wirtschaftlicher Leistungsaustausch zugrunde. Ein dennoch bestehender äußerer Zusammenhang mit bestimmten beruflichen Leistungen ist unschädlich, wenn mit der Auszeichnung nicht in erster Linie eine bestimmte berufliche Leistung des Preisträgers, sondern seine Persönlichkeit geehrt werden soll. Dies kann ausnahmsweise auch angenommen werden, wenn zwar eine bestimmte Leistung Anlass für die Preisverleihung war, zur Auswahl des Preisträgers jedoch dessen Gesamtpersönlichkeit oder Gesamtschaffen entscheidend beigetragen haben (so insbesondere bei der Vergabe des Nobelpreises).

Gegen eine solche vorrangige Ehrung der Persönlichkeit und des Lebenswerks des Preisträgers können nachstehende Umstände sprechen:

- junges oder nur mittleres bzw. noch nicht fortgeschrittenes Alter des Preisträgers,

- die Auszeichnung nur bestimmter Leistungen oder Werke,

- das Fehlen einer Würdigung von Gesamtschaffen oder Gesamtwerk mit einer umfassenden Wertung aller Hauptwerke oder Hauptleistungen und aller von innen ausgestrahlten Wirkungen,

- die mehrmalige Auszeichnung mit dem gleichen Preis.

3. Aus den vorstehenden, in der finanzgerichtlichen Rechtsprechung entwickelten Abgrenzungskriterien lassen sich folgende Handlungsempfehlungen ableiten:

\section{a) Bezeichnung der Preise}

Als Indiz für die steuerliche Einordnung der Preise und der damit verbundenen finanziellen Dotation kann zunächst die Bezeichnung des Preises selbst dienen. So deutet z.B. die Bezeichnung „Hamburger Lehrpreis“ darauf hin, dass der Preis vorrangig und untrennbar auf die von Hochschullehrern im Rahmen ihrer Beschäftigung an Hochschulen erbrachten besonderen Leistungen in der "Lehre“ anknüpft und damit steuerpflichtig ist. Daher sollten bei der Benennung des jeweiligen Preises die damit verbundenen steuerlichen Implikationen berücksichtigt werden und ggf. eine bewusst weite Bezeichnung gewählt werden, die - wenn dies zutrifft - nicht auf einen speziellen Tätigkeitsbereich bzw. eine bestimmte Leistung des Preisträgers hinweist.

b) Öffentliche Darstellung des Preises

Auch der öffentlichen Darstellung des Preises kommt eine wesentliche Indizwirkung zur steuerlichen Einordnung der Preisgelder zu. Wird ein bestimmter Tätigkeitsbereich auch in der Auslobung, den Ausschreibungsbedingungen, in Preisverlei-
hungs-Schreiben, -Reden und Pressemeldungen hervorgehoben, liegt die Annahme nahe, dass nicht die Persönlichkeit des Preisträgers insgesamt, sondern nur eine bestimmte Leistung geehrt werden soll und es sich daher um eine rein berufsbezogene Auszeichnung handelt. Gerade der Inhalt der Ausschreibung verdeutlicht, wem und wofür die Auszeichnung überhaupt zu Gute kommen kann.

Somit sollte bei der öffentlichen Darstellung bereits von Anfang an darauf geachtet werden, dass der Preis - so dies der Fall ist - nicht in Bezug zu einer bestimmten Leistung steht, sondern zu der auszuzeichnenden Person als Ganzes. Nicht das Werk, sondern die Persönlichkeit des Preisträgers sollte in diesen Fällen im Vordergrund der Ehrung stehen und dies sollte auch nach außen hin kundgetan werden. In entsprechenden Verlautbarungen über die Verleihung des Preises sollte man sich in diesen Fällen nicht auf die Hervorhebung bestimmter Leistungen des Preisträgers beschränken; vielmehr bietet es sich an, weiter auszuholen und über das Dienstverhältnis des Preisträgers hinaus sein gesamtes Tätigkeitsfeld mit seinem weit umfangreicheren und ebenfalls bedeutenden Gesamtschaffen lobend zu erwähnen. Dass hierbei auch auf einzelne besonders gelungene Verdienste Bezug genommen wird, ist unschädlich, solange der Schwerpunkt der Darstellung noch auf der Gesamtpersönlichkeit des Preisträgers liegt.

Muss zur Erzielung des Preises ein besonderes Werk geschaffen oder eine spezielle Leistung erbracht worden sein bzw. wird der Preis gerade wegen einer solchen Leistung vergeben, so liegt regelmäßig eine für den jeweiligen Preisträger steuerpflichtige Zuwendung vor. Dies ist insbesondere bei wissenschaftlichen oder künstlerischen Leistungen, die in Ausübung der jeweiligen beruflichen Tätigkeit des Preisträgers erbracht werden, regelmäßig der Fall.

Um Preisträger auf die eventuelle Steuerpflicht vorzubereiten, sollten sie im Zusammenhang mit der Preisverleihung von der jeweiligen Einrichtung darauf hingewiesen werden, dass das Preisgeld möglicherweise der Versteuerung durch den Preisträger unterliegt und nicht vorschnell bzw. ohne Bildung von Rücklagen ausgegeben werden sollte.

\section{Korrespondenzadresse:}

Dr. iur. Albrecht Wienke

Wienke \& Becker - Köln, Sachsenring 6, 50677 Köln

Awienke@Kanzlei-WBK.de 
Bitte zitieren als

Kuball LK, Wienke A. Müssen Preisgelder versteuert werden? In der Regel ja! GMS Mitt AWMF. 2015;12:Doc7.

DOI: 10.3205/awmf000306, URN: urn:nbn:de:0183-awmf0003069

Artikel online frei zugänglich unter

http://www.egms.de/en/journals/awmf/2015-12/awmf000306.shtml
Eingereicht: 20.10.2015

Veröffentlicht: 21.10 .2015

Copyright

C2015 Kuball et al. Dieser Artikel ist ein Open-Access-Artikel und steht unter den Lizenzbedingungen der Creative Commons Attribution 4.0 License (Namensnennung). Lizenz-Angaben siehe http://creativecommons.org/licenses/by/4.0/. 